\title{
Performance Analysis of Digital Fir Filter Using Bat Optimization
}

\author{
Harmandeep Kaur ${ }^{1}$, Dr. Manjit Verma ${ }^{2}$ \\ School of Engineering (Electronics and Communication Engineering) \\ RIMT University, Mandi Gobindgarh, Punjab, India \\ bainsharman94@gmail.com,manjitverma@rimt.ac.in
}

\begin{abstract}
In this paper, filter design procedure is analysed for the designing of finite impulse response (FIR) filter with wanted parameter details using two optimization techniques known as Bat and Particle Swarm Optimization (PSO) optimization algorithms. Earlier optimization techniques were not efficient in solving non-differential functions as those for FIR filters and these stuck on local optimal solution. Therefore, in this PSO and BAT optimization algorithms are used to overcome this problem. These are based on the population method where a group of individuals are considered and their parameters are analysed and varied to reach at the optimal result. PSO works in correspondence to the swarm of birds whereas bat is based on the behaviour of bats and their echolocation property. Using these algorithms, the optimal impulse response coefficients of the different FIR filters are determined to meet their ideal response. Simulative results of both these techniques are compared on the basis of their pass band and stop ripples. These results demonstrate that the Bat is slightly better than PSO.
\end{abstract}

Keywords: FIR filter; PSO; Bat algorithm

\section{INTRODUCTION}

A filtering is a process by which required frequency components of the signal are made to pass through the circuit and unwanted are rejected. It changes the frequency response of any signal in correspondence to the system requirements. Depending upon the type of the signal, filtering can be analog and digital. Analog filters deals with analog signals and are made up of analog components whereas digital filters are programmed on a chip and operates on digital signals. With the various advancements in integrated technology, digital filters are getting much popularity due to well-known merits of digital field in spite of their higher cost which is due to the system complexity. Digital filters are further categorized into Finite Impulse Response (FIR) filters and Infinite Impulse Response (IIR) filters. FIR filters also known as non-recursive filters have finite duration of their impulse response. It has only zeroes and termed as all-zero filters. Whereas IIR filters contains both poles and zeroes and has infinite duration of impulse response. Due to the linearity and stability of the output, FIR filters are the preferred choice among the digital filters. Apart from this, filters are also divided on the basis of the selection of different frequency bands as low pass, high pass, band pass, and band stop filters [1] [2].

Designing of a filter requires its parameters to be selected appropriately in order to from a required frequency response. These parameters are pass band frequency, stop band frequency, bass band and stop band ripples, filter order etc. Since years, different design approaches are considered by different researchers and proved their efficiency for designing FIR filters such as windows design method [1] [3] [4] [5], frequency sampling technique [1] [4] [5] [6], and optimal design or weighted least squares [1] [4] [5]. Earlier windows technique was one of the favourable methods for designing of these filters but loses its interest due to the generation of non-optimal solution and moreover, in windows method, a designer has to compromise on certain design parameters. Therefore, to overcome these disadvantages, FIR filters were designed by using optimization methods which can approximately generates an ideal filter. An optimization is a process in which best possible variables are selected form a given variables under desired conditions. Different design algorithms such as Parks McClellan algorithm [7], Genetic Algorithm (GA) [8], Particle Swarm Optimization (PSO) [8] [9] [10], Differential Evolution (DE) [10] [11], Artificial Bee Colony Optimization (ABC) [12], Teaching Learning Based Optimization (TLBO) [13] [14], Cat Swarm optimization [14] and Cuckoo Search algorithm (CS) [15] have been used in the design procedure of FIR filters. Yang [16] developed a new algorithm known as Bat optimization algorithm which is based on the behaviour of bats and utilises their echolocation property. Using this echolocation phenomenon, they find out the distance of their food. Severino et al. presented the design algorithm for low pass FIR filter using PSO and Bat optimization technique and Bat optimization proves to be best among PSO and Parks McClellan [17].

Nowadays, Bat algorithm is considered an appropriate optimization technique for solving majority of the optimization problems. It is being used in almost every field of engineering, such as optimization [16] [18], image processing [19] [20], data mining, feature selection, fuzzy logic [21] [22], artificial neural networks and many more. As per the literature review, it is not been used much for optimizing FIR filter parameters. In this paper, BAT optimization algorithm has been used to 
design the FIR band pass filter. The simulation results based on this design is presented here.

The paper is arranged in the following manner. Section 1 presents the introduction. Section 2 describes the design procedure. In Section 3 and 4, PSO and Bat optimization technique are discussed. Section 5 presents the simulation results obtained by the technique. Finally, Section 6 concludes the paper.

\section{DESIGN PROCEDURE}

In digital filter design, the main aim is to form favourable magnitude response characteristics in accordance with the given design parameters of filters. Filters are usually designed as per the characteristics of an ideal filter. However ideal filters are non-causal filters and are of infinite duration. So FIR filers are formed by truncating the impulse response of ideal filters and their response characteristics are made comparable to the frequency response characteristics of filter [1] [2] [4]. The frequency response of filter is given as:

$\mathbf{H}_{\mathbf{d}}\left(\mathrm{e}^{\mathrm{iw}}\right)=\sum_{\mathbf{n}=\mathbf{0}}^{\mathrm{N}} \mathbf{h}(\mathbf{n}) \mathbf{e}^{-\mathrm{iwn}}$

(1)

$\mathbf{n}=\mathbf{0}, \mathbf{1}, \ldots \ldots \ldots \ldots \mathbf{N}$

where $h(n)$ represents the impulse response of filter. $\mathrm{N}$ is the order of filter with $\mathrm{N}+1$ number of coefficients. Filters are also classified according to the selection of the desired frequency components as low pass, high pass, band pass and band stop filters. Low pass filters are termed as the filters which selects the low frequency band and rejects the frequency components more that the cut-off frequency. High pass filters rejects the low frequencies till the cut-off frequency. Band pass and band stop filters have two cut-off frequencies as they pass and stop the desired band of frequencies starting from first cut-off value and ending at the second one. Band pass filters pass that desired band and band stop filters stop the desired band of frequencies. However, the designed filter is not same as that of ideal filter as it have some ripples in pass band as well as stop band which can be in individually specified in some of the design techniques. $\boldsymbol{\delta}_{\mathbf{p}}$ and $\boldsymbol{\delta}_{\mathbf{s}}$ are the ripple magnitudes of pass-band and stop-band respectively

$\delta_{\mathrm{p}}=\max _{\mathrm{wi}}\left|\mathrm{H}\left(\mathrm{w}_{\mathrm{i}}, \mathrm{x}\right)\right|-\min _{\mathrm{wi}}\left|\mathrm{H}\left(\mathrm{w}_{\mathrm{i}}, \mathrm{x}\right)\right|$

$\delta_{\mathrm{s}}=\max _{\mathrm{wi}}\left|\mathrm{H}\left(\mathrm{w}_{\mathrm{i}}, \mathrm{x}\right)\right|$

For wi $€$ pass band

The main objective of this paper is to design an FIR filter with optimal design conditions by using PSO and Bat optimization algorithms. The designed FIR filter is of linear phase and even order and thus the coefficients of this filter come out to be even symmetric which reduces the dimension of our design problem. As only half of the coefficients are optimized using optimization techniques and by using concatenation operation final $\mathrm{N}+1$ coefficient are formed. As seen from the literature, different parameters are optimized by fixing the other parameters. In [15], filter order (N), pass band and stop band ripples are made fixed and varying the other parameters. In Parks and McClellan (PM) algorithm [7], filter order (N), pass band and stop band frequencies $\left(\mathrm{w}_{\mathrm{p}}\right.$ and $\left.\mathrm{w}_{\mathrm{s}}\right)$, and the ratio of $\delta_{\mathrm{p}} / \delta_{\mathrm{s}}$ is fixed. Error function is the main parameter which is considered in almost all optimization problems for designing FIR filters. It is considered to be the minimization objective function of the problem to be solved which needs to be minimized by the application of iteration procedure.

The Parks McClellan algorithm uses the error function [7] as presented in (10).

$\mathbf{E}(\mathbf{w})=\mathbf{G}(\mathbf{w})\left[\mathbf{H}_{\mathbf{d}}\left(\mathbf{w}_{\mathbf{k}}\right)-\mathbf{H}_{\mathbf{i}}\left(\mathbf{w}_{\mathbf{k}}\right)\right]$

where $\mathbf{H}_{\mathbf{d}}\left(\mathbf{w}_{\mathbf{k}}\right)$ is the frequency response of desired filter and $\mathbf{H}_{\mathbf{i}}\left(\mathbf{w}_{\mathbf{k}}\right)$ is the frequency response of ideal filter. $G(w)$ represents the weighting function. The ratio of $\boldsymbol{\delta}_{\mathbf{p}} / \boldsymbol{\delta}_{\mathbf{s}}$ is fixed in this which is the main drawback of this phenomenon. Different literatures deal with the different error functions as presented in [23] [24] [25]. However, every design problem has their merits and demerits. The error function which is used in this paper has the advantage of individually defining the required values for pass band and stop band ripples [8].

$J_{1}=\max _{w \leq \mathrm{w}_{p}}\left(|\mathrm{E}(\mathrm{w})|-\delta_{\mathrm{p}}\right)+\max _{w \geq \mathrm{w}_{s}}\left(|\mathrm{E}(\mathrm{w})|-\delta_{\mathrm{s}}\right)$

\section{PSO OPTIMIZATION ALGORITHM}

Particle swarm optimization algorithm is a swarm based technique based on the swarm of birds or school of fish or any other living creatures that follow the path by learning from their fellow mates. It was initially developed by Kennedy and Eberhart [26] in 1995. After its origin, it has gained very popularity in almost every field of optimization as it has the advantage of not getting trapped in the local optimal solution but solves the problem at a global approach [27]. Every individual varies their parameters in accordance with the group of individuals known as swarm especially position has two values-personal best (pbest) and global best (gbest) where personal best is the best position of individual particle and global best is the best position of that particle among the entire group. Each particle modifies their position and velocity according to the following equations and this is done by following the iterative procedure. The velocity of the particle is updated as per the following equation:

$V_{i}^{(k+1)}=w * V_{i}^{k}+C_{1} * \operatorname{rand}_{1} *\left(\right.$ pbest $_{i}^{k}-$

$\left.S_{i}^{k}\right)+C_{2} * \operatorname{rand}_{2} *\left(\right.$ gbest $\left.^{k}-S_{i}^{k}\right)$

where $V_{i}^{k}$ is the velocity of $\mathrm{i}^{\text {th }}$ particle vector at $\mathrm{k}^{\text {th }}$ iteration; $\mathrm{w}$ is the weighting function; $\mathrm{C}_{1}$ and $\mathrm{C}_{2}$ are the positive weighting factors; rand $_{1}$ and rand $_{2}$ are the random numbers between 0 and $1 ; S_{i}^{k}$ is the current position of $i^{\text {th }}$ particle vector $h(n)$ at $k^{\text {th }}$ 
iteration; pbest ${ }_{i}^{k}$ is the personal best of the $\mathrm{i}^{\text {th }}$ particle at the $\mathrm{k}^{\text {th }}$ iteration; gbest $^{k}$ is the group best of the group at the $\mathrm{k}^{\text {th }}$ iteration.

The position of the particle is updated according to the following equations:

$X_{i}^{(k+1)}=X_{i}^{k}+V_{i}^{(k+1)}$

\subsection{Filter design using PSO algorithm}

PSO is considered to be efficient for solving nondifferential functions like those for FIR filters. Following steps are followed for solving this design problem:

Step 1: Specify the controlling parameters for FIR filters such as pass band and stop band frequencies, order of the filter, pass band and stop band ripples.

Step 2: Specification of PSO parameters such as control parameters of PSO (cognitive and social acceleration values, weighting function and the values for rand1 and rand2), maximum number of iterations and size of population.

Step 3: Initialize the positions of the particle in a population according to the coefficients of filter and also define the value of velocity.

Step 4: Calculate the fitness value for each particle using "eq. (5)".

Step 5: Update the positions and velocities of all the particles in accordance with "eqs. (6) and (7).

Step 6: Measure the fitness from the updated value of velocity and position.

Step 7: Check if the updated fitness value of the personal best is less than the earlier value of fitness. If yes mark it to be best value otherwise condition for the number of maximum iteration is viewed. If yes program is terminated otherwise again start from step 5 .

Step 8: Look for the updated fitness value in accordance with the global value. If it comes out to be less than previous value than stop the process otherwise check whether maximum number of iteration is reached. If yes terminate the program and if not repeat from the step 5 .

\section{BAT OPTIMIZATION ALGORITHM}

Bat optimization is a search algorithm depending upon the behaviour of bats and their echolocation capability. It is proposed by Yang [16] and works according to the search behaviour of bats for their food. This technique is almost similar to PSO but senses the distance using echolocation property and takes advantage of frequency equation. To utilise this algorithm for any optimization problem, initialised values for velocity, position, minimum frequency, wavelength and loudness values are set to find out the target.

\section{Bat Motion and variations of loudness and pulse} rates

Each bat has its initialized velocity $v_{i}$, position $x_{i}$ and pulse frequency $f_{i}$ in a solution space. The new velocity position $v_{i}^{t}$ and position $x_{i}^{t}$ of the bats are updated at every time step $t$ in accordance with the velocity and position equations as follows:

$\mathrm{f}_{\mathrm{i}}=\mathrm{f}_{\text {min }}+\left(\mathrm{f}_{\text {max }}-\mathrm{f}_{\text {min }}\right) \beta$

$v_{i}^{t}=v_{i}^{t-1}+\left(x_{i}^{t-1}-x^{*}\right) f \quad I$

$\mathrm{x}_{\mathrm{i}}^{\mathrm{t}}=\mathrm{x}_{\mathrm{i}}^{\mathrm{t}-1}+\mathrm{v}_{\mathrm{i}}^{\mathrm{t}}$

$\mathrm{x}_{\text {new }}=\mathrm{x}_{\text {old }}+\varepsilon \mathrm{A}^{\mathrm{t}}$

$\mathrm{A}_{\mathrm{i}}^{\mathrm{t}+1}=\alpha \mathrm{A}_{\mathrm{i}}^{\mathrm{t}}$

$\mathrm{r}_{\mathrm{i}}^{\mathrm{t}+1}=\mathrm{r}_{\mathrm{i}}^{0}[1-\exp (-\gamma \mathrm{t})]$

\subsection{Filter design using Bat algorithm}

For designing FIR filters using Bat algorithm, following steps are implemented:

Step 1: Determine the controlling parameters for FIR filter such as pass band and stop band frequencies, order of the filter, pass band and stop band ripples.

Step 2: Specify he parameters for Bat optimization such as loudness, pulse rate value, the frequency range to determine the scaling, maximum number of iterations and population size.

Step 3: Initialize the arrays for the frequency and velocity values.

Step 4: Calculate the fitness value for each individual using "eq. (5)".

Step 5: Update the frequency, velocities and positions of all the individuals in accordance with "eqs. (8), (9) and (10).

Step 6: Check if random number generated is greater than pulse rate value, if yes then select the solution from the best ones and generate a local solution around this using "eq. (11)" otherwise generate a local solution by randomly selecting a solution.

Step 7: Measure the fitness from these updated values. Update the new solutions otherwise repeat the process from step 5. Also update the value of loudness and pulse rate.

Step 8: Generate the filter coefficients using these updated solutions by selecting the best bat among the entire group.

\section{SIMULATIVE RESULTS}

This section illustrates the simulative results performed on MATLAB software for the designing of FIR band pass filter. The filter parameters are as: filter order $=40$ thus filter coefficients $=41$, sampling frequency $=1 \mathrm{~Hz}$, frequency samples = 512. For band pass upper and lower cut off frequencies (normalised) are 0.3 and 0.7 respectively. The number of iterations considered for the optimization problem to achieve the optimal solution $=100$. Table 1 shows the parameters for the PSO and Bat algorithm. Table 2 shows the optimized filter coefficients for both PSO and Bat optimization. These coefficients are obtained for filter order 40 thus generating number of coefficients to be 41. As the filter is even symmetric, only 21 coefficients are formed and rest are same to the previous ones. 
International Journal of Research in Advent Technology, Vol.7, No.4, April 2019

E-ISSN: 2321-9637

Available online at www.ijrat.org

Table 1: Parameters for the PSO and Bat algorithm

\begin{tabular}{|l|l|l|}
\hline Parameters & PSO & Bat \\
\hline Population Size & 20 & 20 \\
\hline Max.Iteration Cycle & 100 & 100 \\
\hline $\mathrm{C}_{1}, \mathrm{C}_{2}$ & 2,2 & \\
\hline $\mathrm{V}_{1}{ }^{\min }, \mathrm{v}_{1}{ }^{\max }$ & 0,1 & \\
\hline $\mathrm{W}$ & 1 & \\
\hline $\mathrm{A}$ & & 0.5 \\
\hline $\mathrm{R}$ & & 0.5 \\
\hline $\mathrm{F}_{\min }, \mathrm{F}_{\max }$ & & 0,2 \\
\hline $\begin{array}{l}\text { Limits of filter } \\
\text { coefficients }\end{array}$ & 0,2 & 0,2 \\
\hline
\end{tabular}

Table 2: Optimized coefficients of the FIR BP filter of order 40

\begin{tabular}{|c|c|c|}
\hline & PSO coefficients & BAT coefficients \\
\hline$h(1)=h(41$ & $\begin{array}{l}- \\
0.001214369204 \\
505\end{array}$ & $\begin{array}{l}- \\
0.000000000000 \\
000\end{array}$ \\
\hline$h(2)=h(40$ & $\begin{array}{l}0.001522268564 \\
581\end{array}$ & $\begin{array}{l}0.000000000000 \\
000\end{array}$ \\
\hline$h(3)=h(39$ & $\begin{array}{l}0.002973535838 \\
728\end{array}$ & $\begin{array}{l}0.003455252881 \\
205\end{array}$ \\
\hline$h(4)=h(38$ & $\begin{array}{l}- \\
0.001645226601 \\
158\end{array}$ & $\begin{array}{l}- \\
0.000000000000 \\
000\end{array}$ \\
\hline$h(5)=h(37$ & $\begin{array}{l}- \\
0.000735603523 \\
839 \\
\end{array}$ & $\begin{array}{l}- \\
0.003933584849 \\
155 \\
\end{array}$ \\
\hline$h(6)=h(36$ & $\begin{array}{l}- \\
0.001883697667 \\
381\end{array}$ & $\begin{array}{l}- \\
0.000000000000 \\
000\end{array}$ \\
\hline$h(7)=h(35$ & $\begin{array}{l}- \\
0.009748958753 \\
003\end{array}$ & $\begin{array}{l}- \\
0.007221104373 \\
597\end{array}$ \\
\hline$h(8)=h(34$ & $\begin{array}{l}0.006430359196 \\
770\end{array}$ & $\begin{array}{l}0.000000000000 \\
000\end{array}$ \\
\hline$h(9)=h(33$ & $\begin{array}{l}0.015163993476 \\
709\end{array}$ & $\begin{array}{l}0.020114518991 \\
526\end{array}$ \\
\hline $\begin{array}{l}h(10)=h(3 \\
2)\end{array}$ & $\begin{array}{l}- \\
0.002567195959 \\
388\end{array}$ & $\begin{array}{l}- \\
0.000000000000 \\
000\end{array}$ \\
\hline $\begin{array}{l}\mathrm{h}(11)=\mathrm{h}(3 \\
1)\end{array}$ & $\begin{array}{l}0.010132039484 \\
216\end{array}$ & $\begin{array}{l}0.000000000000 \\
000\end{array}$ \\
\hline $\begin{array}{l}h(12)=h(3 \\
0)\end{array}$ & $\begin{array}{l}- \\
0.009569681967 \\
778 \\
\end{array}$ & $\begin{array}{l}- \\
0.000000000000 \\
000\end{array}$ \\
\hline $\begin{array}{l}h(13)=h(2 \\
9)\end{array}$ & $\begin{array}{l}- \\
0.052623777266 \\
360\end{array}$ & $\begin{array}{l}- \\
0.051731808303 \\
021\end{array}$ \\
\hline $\begin{array}{l}h(14)=h(2 \\
8)\end{array}$ & $\begin{array}{l}0.012823827125 \\
964\end{array}$ & $\begin{array}{l}0.000000000000 \\
000\end{array}$ \\
\hline $\begin{array}{l}h(15)=h(2 \\
7)\end{array}$ & $\begin{array}{l}0.036064140137 \\
315\end{array}$ & $\begin{array}{l}0.050643023710 \\
857\end{array}$ \\
\hline $\begin{array}{l}h(16)=h(2 \\
6)\end{array}$ & $\begin{array}{l}0.002703712487 \\
528\end{array}$ & 0 \\
\hline $\begin{array}{l}\mathrm{h}(17)=\mathrm{h}(2 \\
5)\end{array}$ & $\begin{array}{l}0.098874631953 \\
592\end{array}$ & $\begin{array}{l}0.085504061477 \\
255\end{array}$ \\
\hline
\end{tabular}

\begin{tabular}{|l|l|l|}
$\mathrm{h}(18)=\mathrm{h}(2$ & - & - \\
$4)$ & 0.018564998047 & 0.000000000000 \\
412 & 000 \\
\hline $\mathrm{h}(19)=\mathrm{h}(2$ & - & - \\
$3)$ & 0.289544756381 & 0.296517071230 \\
137 & 397 \\
\hline $\begin{array}{l}\mathrm{h}(20)=\mathrm{h}(2 \\
2)\end{array}$ & $\begin{array}{l}0.011207972060 \\
511\end{array}$ & 0 \\
\hline $\mathrm{h}(21)$ & $\begin{array}{l}0.381081527852 \\
430\end{array}$ & $\begin{array}{l}0.400813827342 \\
926\end{array}$ \\
\hline
\end{tabular}

Figure 1 shows the comparison of the normalized magnitude responses of both PSO and Bat optimization technique. Figure 2 depicts the $\mathrm{dB}$ plot for FIR pass band ripple. Figures 3 and 4 represents the pass band and stop band ripples for FIR band pass filter. In figure 5, comparison of the convergence profile i.e. the error function is shown for both the optimization techniques. The minimum fitness function for PSO is 94.162448726144376 and for Bat, it is -2.0073 . This clearly shows that the error function for Bat comes out to be less than PSO depicting that it can best approximate the ideal filter characteristics. Till the iteration number 48, PSO value was less than Bat but at higher iterations, PSO value goes slightly higher than bat.

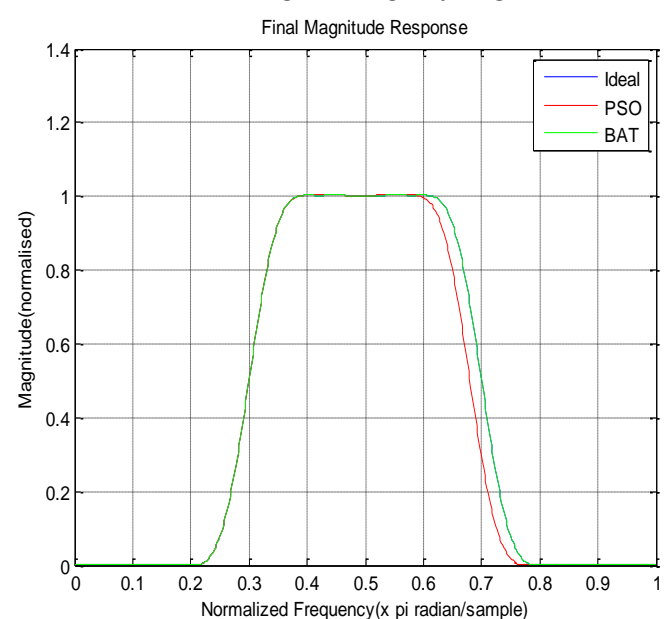

Figure 1: Normalised plot for FIR filter

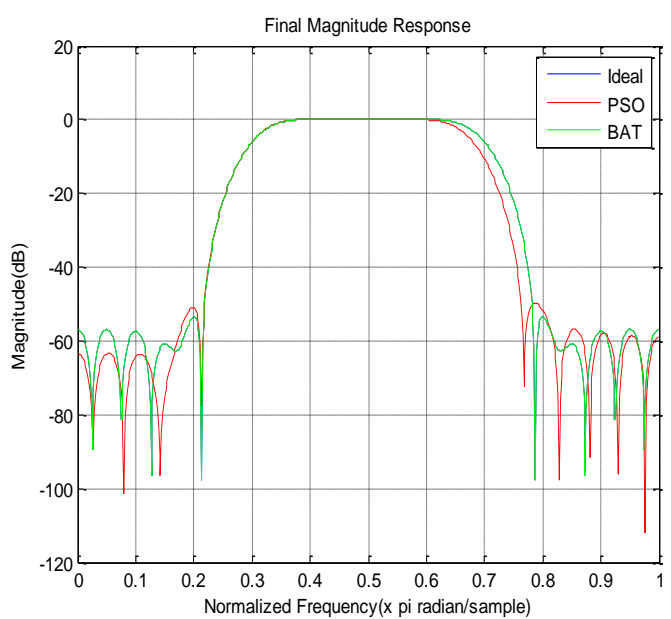

Figure $2: \mathrm{dB}$ plot for FIR filter 


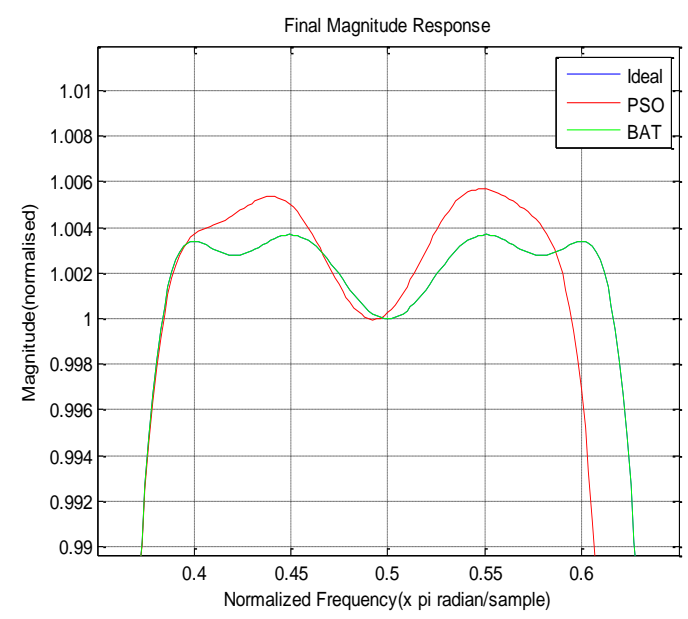

Figure 3 : Normalised Pass band ripples

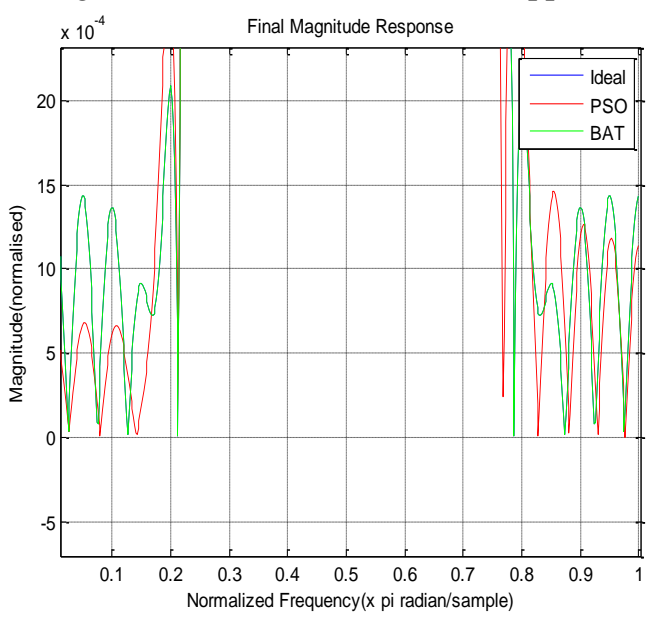

Figure 4: Normalised Stop band ripples

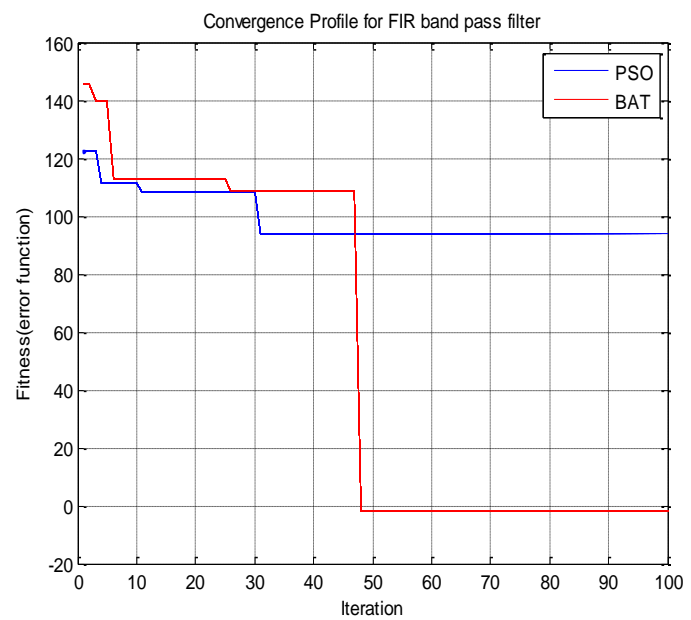

Figure 5: Comparison of convergence profile for PSO and Bat

\section{CONCLUSION}

In this paper, FIR band pass filter is designed using Bat and PSO algorithms. As per the output variable and figures, both the techniques were found efficient for this processing. Earlier optimization techniques could not appropriately design an FIR filter which is a multi-modal problem, as those techniques usually struck on the local optimal solution. In order to overcome that PSO and BAT optimization algorithms are used in this paper and efficiently proved their results. The magnitude response generated are very feasible and also the pass band and stop band ripples are very less comparable to the earlier techniques. Maximum pass band ripple for Bat is approximately equals to $1.0039 \mathrm{~dB}$ which is quite a low value and similarly for PSO it is 1.0057 . The value of error function is also not very high which is equals to 94.162448726144376 for PSO and for Bat, it is 2.0073 making the techniques competitive to other optimization methods.

\section{BIBLIOGRAPHY}

[1] T. W. Parks and C. S. Burrus, Digital Filter Design.: New York: Wiley, 1987.

[2] A. Antoniou, Digital filters: analysis and design.: New York: McGraw Hill.

[3] F. J. Harris, "On the Use of Windows for Harmonic Analysis with the Discrete Fourier Transform," Proc. IEEE, vol. 66, no. 1, 1978.

[4] L. R. Rabiner and B. Gold, Theory and Applications of Digital Signal Processing.: New Jersey: Prentice-Hall, 1975.

[5] J. G. Proakis and D. G. Manolakis, Digital Signal Processing-Principles, Algorithms and Applications, Fourth edition ed., Pearson, Ed. United States: Prentice-Hall, 1997.

[6] L. R. Rabiner, B. Gold, and C. A. McGonegal, "An approach to the Approximation Problem for Nonrecursive Digital Filters," IEEE Trans. Audio and Electroacoustics, vol. AU-18, pp. 83-105, June 1970.

[7] T. W. Parks and J. H. McClellan, "Chebyshev Approximation for Non-recursive Digital Filters with Linear Phase," IEEE Transactions on Circuit Theory, vol. 19, no. 2, pp. 189-194, 1972.

[8] J. I. Ababneh and M. H. Bataineh, "Linear phase FIR filter design using particle swarm optimization and genetic algorithms," Digital Signal Processing, vol. 18, no. 4, pp. 657-668, 2008.

[9] A. K. Jatana and D. S. Sidhu, "Design of Digital FIR High Pass Filter Using Particle Swarm Optimization (PSO) Technique," International Journal of Scientific Research Engineering and Technology, vol. 4, no. 5, pp. 472-479, 2015.

[10] H. Sidhu, R. Kaur, and B. Singh, "Design of FIR Band-pass Digital Filter using Heuristic Optimization Technique: A Comparison," in International Conference on Communication, Control and Intelligent Systems, Mathura, 2015, pp. 244-249.

[11] S. R. Kotha and S. K. Sahoo, "An approach 
for FIR filter coefficient optimization using differential evolution algorithm," International Journal of Electronics and Communications, vol. 69, no. 1, pp. 101-108, 2014.

[12] D. Ji, "The Application of Artificial Bee Colony (ABC) Algorithm in FIR Filter Design," in 12th International Conference on Natural Computation, Fuzzy Systems and Knowledge Discovery, 2016, pp. 663-667.

[13] R. V. Rao, V. J. Savsani, and D. P. Vakharia, "Teaching-learning-based optimization: A novel method for constrained mechanical design optimization problems," ComputerAided Design, vol. 43, no. 3, pp. 303-315, 2011.

[14] S. K. Saha, S. P. Ghoshal, R. Kar, and D. Mandal, "Cat Swarm Optimization algorithm for optimal linear phase FIR filter design," ISA Transactions, vol. 52, no. 6, pp. 781-794, 2013.

[15] I. Sharma, B. Kuldeep, A. Kumar, and V. K. Singh, "Performance of swarm based optimization techniques for designing digital FIR filter: A comparative study," An International Journal of Engineering Science and Technology, vol. 19, no. 3, pp. 1564-1572, 2016.

[16] X. S. Yang, "A New Metaheuristic BatInspired Algorithm," Nature Inspired Cooperative Strategies for Optimization, vol. 284, no. 2, pp. 65-74, 2010.

[17] A. G.V. Severino, L. L.S. Linhares, and F. M.U. DeAraujo, "Optimal Design of Digital Low Pass Finite Impulse Response Filter using Particle Swarm Optimization and Bat Algorithm," in 12th International Conference on Informatics in Control, Automation and Robotics, July 2015, pp. 207-214.

[18] X. S. Yang and S. Deb, "Eagle strategy using L'evy walk and firefly algorithms for stochastic optimization," Studies in Computational Intelligence, vol. 284, pp. 101111,2010

[19] E. M. Abdel-Rahman, A. R. Ahmad, and S. Akhtar, "A metaheurisic bat- inspired algorithm fJor full body human pose estimation," in Ninth Conference on Computer and Robot Vision, 2012, pp. 369-375.
[20] Z. Y. Du and B. Liu, "Image matching using a bat algorithm with mutation," Applied Mechanics and Materials, vol. 203, no. 1, pp. 88-93, 2012.

[21] T. A. Lemma and F. Bin Mohd Hashim, "Use of fuzzy systems and bat algorithm for exergy modelling in a gas turbine generator," in IEEE Colloquium on Humanities, Science and Engineering, 5-6 Dec. 2011, pp. 305-310.

[22] A. L. Tamiru and F. M. Hashim, "Application of bat algorithm and fuzzy systems to model energy changes in a gas turbine," Studies in Computational Intelligence, vol. 427, pp. 685719, 2013.

[23] N. Karaboga and B. Cetinkaya, "Design of digital FIR filters using differential evolution algorithm," Circuits, Systems, and Signal Processing, vol. 25, no. 5, pp. 649-660, 2006.

[24] G. Liu, Y. X. Li, and G. He, "Design of digital FIR filters using differential evolution algorithm based on reserved gene," in IEEE Conference on Evolutionary Computation, Barcelona, Spain, July 2010, pp. 1-7.

[25] M. Najjarzadeh and A. Ayatollahi, "FIR digital filters design: particle swarm optimization utilizing LMS and minimax strategies," in IEEE International Symposium on Signal Processing and Information Technology, Sarajevo, Bosnia and Herzegovina, December 2008, pp. 129-132.

[26] J. Kennedy and R. Eberhart, "Particle swarm optimization," in Proc. IEEE International Conference on Neural Networks, Perth, Australia, 1995, pp. 1942-1948.

[27] J. Kennedy and R. Eberhart, "A new optimizer using Particle Swarm Theory," in Proc. IEEE Sixth International Symposium on Micro Machine and Human Science, Nagoa, Japan, 1995, pp. 39-43. 